\title{
Carcinoma renal de los conductos colectores de bajo grado en un paciente en tratamiento con hemodiálisis
}

\author{
Martín Martín S, De Castro Olmedo C, Torrecilla García-Ripoll JR, García Lagarto E, \\ Alonso Menéndez S, Fernández del Busto E.
}

Hospital Clínico Universitario. Valladolid.

Actas Urol Esp. 2008;32(5):559-562

\section{RESUMEN}

\section{CARCINOMA RENAL DE LOS CONDUCTOS COLECTORES DE BAJO GRADO EN UN PACIENTE EN TRATAMIENTO CON HEMODIÁLISIS}

Los pacientes en tratamiento con diálisis por enfermedad renal en estadio terminal tienen un riesgo aumentado de padecer varios tipos de cáncer, especialmente de riñón y del tracto urinario.

Presentamos el caso de un varón de 56 años en tratamiento con hemodiálisis. En una ecografía de control se apreció una imagen compatible con un quiste complicado de unos dos centímetros a nivel del polo inferior de riñón izquierdo, que es confirmada mediante TC y RM abdominal.

Se practicó una nefrectomía izquierda por vía laparoscópica. El diagnóstico histológico fue de carcinoma renal de los conductos colectores de bajo grado, una variante infrecuente de tumor renal.

Palabras Clave: Carcinoma de los conductos colectores. Bajo grado. Diálisis.

\section{ABSTRACT}

LOW-GRADE COLLECTING DUCT CARCINOMA OF THE KIDNEY IN A PATIENT TREATED BY DIALYSIS.

Patient treated by dialysis for end-stage renal disease are at increased risk of many cancers, especially those of kidney and urinary tract. A 56 years old man who undergoing chronic dialysis, presented in ultrasonography a $2 \mathrm{~cm}$ complex cystic mass in the left kidney what was confirmed by computed tomography and magnetic resonance.

A laparoscopic left nephrectomy was performed. Histological examination showed a low grade collecting duct carcinoma of the kidney. An infrequent variant of kidney tumor.

Keywords: Collecting duct carcinoma. Low grade. Dialysis.

$\mathrm{L}_{\mathrm{s}}$ os pacientes en hemodiálisis crónica pueden presentar quistes renales múltiples en una proporción elevada, conociéndose como enfermedad renal quística adquirida, y además desarrollar lesiones benignas y malignas en sus riñones naturales ${ }^{1,2}$.

El potencial maligno de los tumores renales en pacientes con fracaso renal crónico es bajo, ya que se trata de neoformaciones bien diferenciadas, de pequeñas dimensiones y curso asintomático ${ }^{1}$, tal como sucedió en el enfermo que presentamos. Lo particular del caso fue que en el estudio histológico de la tumoración renal nos encontramos un carcinoma renal de los túbulos colectores de Bellini, una variante que supone el 1-3\% de todos los tumores renales $^{3}$.

\section{CASO CLÍNICO}

Paciente de 56 años de edad, con antecedentes personales de insuficiencia renal crónica de etiología no filiada (probable nefroangioesclerosis) en tratamiento con hemodiálisis, hipertensión arterial, cardiopatía isquémica y prostatismo, que ingresa en nuestro Servicio procedente del Servicio de Nefrología, por el hallazgo casual de una lesión quística en polo inferior de riñón izquierdo en una ecografía.

El paciente se encuentra asintomático, con la exploración, la analítica general y la radiografía simple del aparato urinario dentro de la normalidad.

Una ecografía abdominal realizada en el contexto de su insuficiencia renal crónica muestra unos 
riñones de pequeño tamaño, con mala diferenciación córtico-medular y un adelgazamiento de la cortical. En el riñón izquierdo se evidencia una lesión quística de pequeño tamaño, con discreto contenido ecogénico en su interior, compatible con un quiste complicado (Fig. 1).

Ante los hallazgos encontrados, se decide realizar un TC abdominal y una resonancia magnética, donde se aprecia, en polo inferir de riñón izquierdo, una imagen nodular de $2 \mathrm{~cm}$. de diámetro con la misma señal que el parénquima renal en todas las secuencias. (Fig. 2).

Tras estudios preoperatorios el paciente es intervenido quirúrgicamente, llevando a cabo una nefrectomía izquierda laparoscópica.

El estudio anatomopatológico demostró la presencia de una lesión nodular sólida, pardo-amarillenta, bien delimitada, que mide $2 \times 1.7 \mathrm{~cm}$ y que está localizada en la zona corticomedular sin alcanzar la superficie externa. Histológicamente se observa una proliferación epitelial muy bien delimitada del parénquima renal adyacente, la cual se muestra constituida por túbulos revestidos de células epiteliales cuboideas o prismáticas dispuestas en una sola hilera. Estas células muestran un moderado isomorfismo y una escasa capacidad mitótica (Fig. 3). Inmunofenotípicamente las células epiteliales proliferantes son positivas a los marcadores AE1-AE3 (Fig. 4) y EMA. La neoformación se extiende comprimiendo la cortical y la pelvis

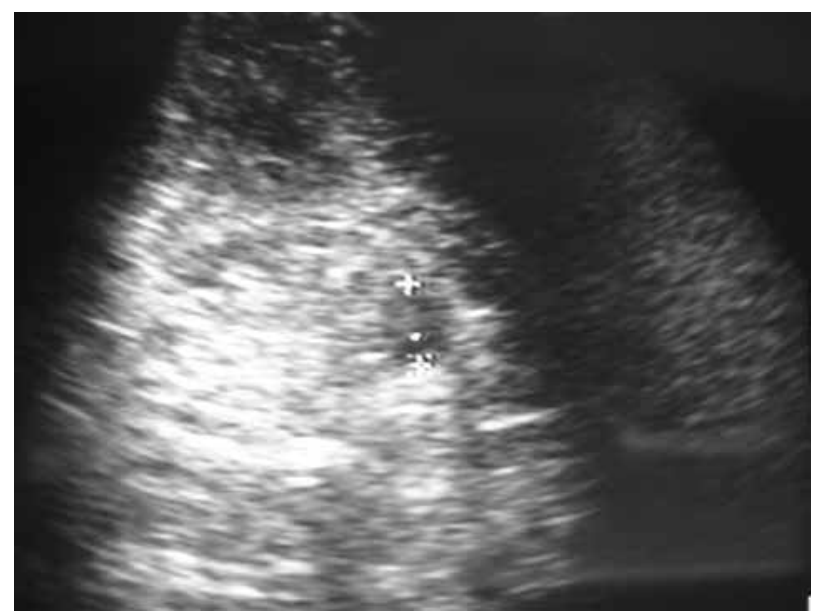

FIGURA 1. La ecografía nos muestra en el riñón izquierdo una imagen quística de pequeño tamaño con discreto contenido ecogénico en su interior compatible con un quiste complicado.

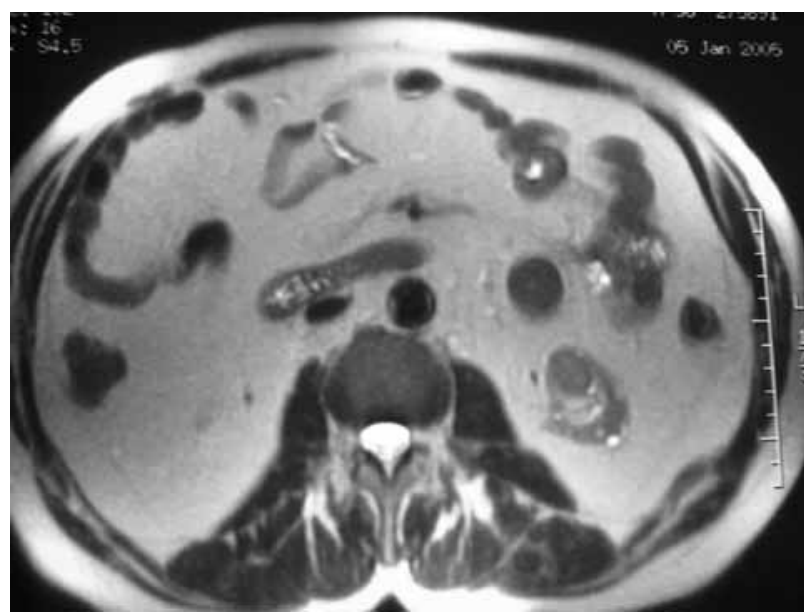

FIGURA 2: En la resonancia magnética se observa una imagen nodular de $2 \mathrm{~cm}$. de diámetro con la misma señal que el parénquima renal en todas las secuencias.

renal. Ni la cápsula renal ni los vasos renales se ven afectados. Ante los hallazgos citológicos, histológicos e inmunohistoquímicos el diagnóstico final fue de carcinoma de bajo grado de los conductos colectores.

El paciente fue dado de alta 8 días después de la cirugía. En el postoperatorio inmediato presentó un cuadro de íleo paralítico que se recuperó tras la aplicación de medidas conservadoras. La evolución posterior fue favorable, sin objetivarse signos de recidiva tumoral ni metástasis tras 15 meses de seguimiento.

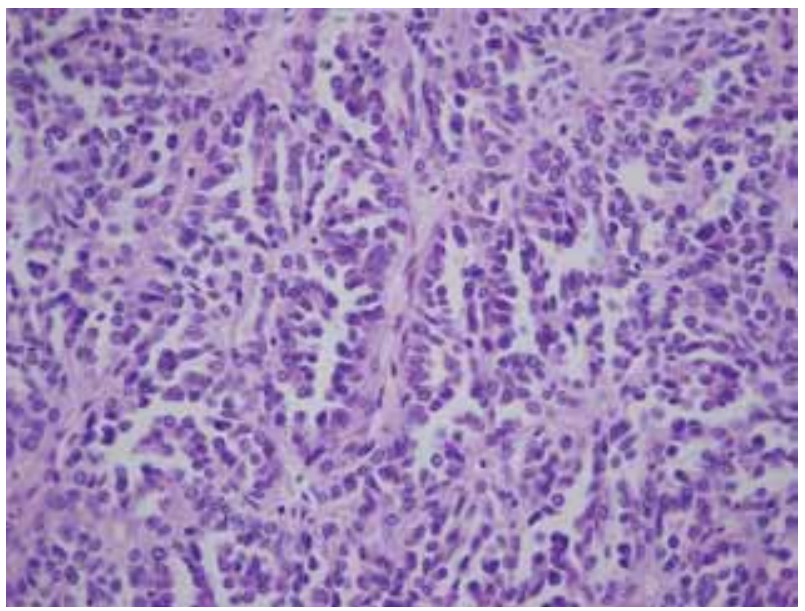

FIGURA 3: Neoformación epitelial constituida por túbu los revestidos por una hilera de células epiteliales monomorfas, con morfologia en tachuela y escasas mitosis. (HE 40x) 


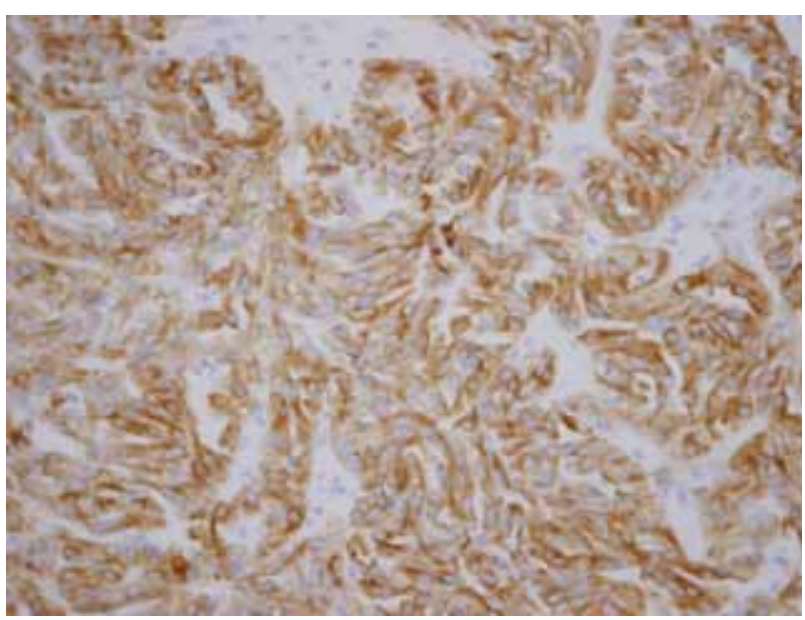

FIGURA 4: Se observó positividad difusa a nivel de membrana citoplasmática de las células tumorales para citoqueratinas AE1-AE3. (AE1-AE3 40x) PIE DE PÁGINA DE para citoqueratinas AE1-AE3. (AE1-AE3 40x)

\section{DISCUSIŌN}

El carcinoma renal de los conductos colectores de Bellini (CRCC) constituye una variante poco frecuente de tumor renal $(1-3 \%)^{3}$, que muestra unas características clínicas, histológicas e inmunohistoquímicas diferentes del carcinoma de células renales. Son tumores que se cree derivan de los conductos colectores de la médula renal. Su origen embriológico, también difiere del de los demás tumores del parénquima renal. Los uréteres, pelvis, cálices, y túbulos colectores derivan de la yema ureteral, la cual se origina a partir del conducto de Wolf. Por otra parte, el blastema metanéfrico es el responsable del desarrollo de los túbulos contorneados, lugar de origen del carcinoma de células renales ${ }^{4,5}$.

Los CRCC fueron reconocidos como entidad clínico-patológica en 1986 por Fleming y Lewi ${ }^{6}$. Epidemiológicamente, existe un predominio masculino (3:1) y se presentan con más frecuencia entre los 50-60 años, aunque los carcinomas de tipo medular, que aparecen relacionados con rasgos hematológicos falciformes y en individuos de raza negra, la media, desciende a los 40 años ${ }^{5}$.

La clínica de estos tumores, al igual que la del resto de los carcinomas de riñón es inespecífica. En nuestro caso el diagnóstico fue de manera incidental, en el estudio ecográfico de un paciente en tratamiento con hemodiálisis.

Clásicamente el CRCC se ha definido como un tumor de alto grado histológico y gran agresividad clínica ${ }^{7}$. Con un elevado riesgo $(72,7 \%)$, de presentarse como enfermedad localmente avanzada y con una probabilidad del 66\% de presentar metástasis ganglionares en pacientes tratados mediante nefrectomía radical ${ }^{8}$. Sin embargo, en 1997, Maclennan et al. ${ }^{9}$ describieron 13 casos similares al nuestro, para los que propusieron el nombre de carcinoma de conductos colectores de bajo grado.

Estos tumores pueden aparecer solos (lo más común), o en formas asociadas a tumores benignos como el adenofibroma metanéfrico o maligno como el carcinoma papilar ${ }^{10}$.

Los CRCC presentan rasgos inmunohistoquímicos peculiares con reactividad a determinadas lecitinas, citoqueratinas de bajo y alto peso molecular, sobre todo estas últimas, y Ulex Europeus. La positividad para el antígeno epitelial de membrana es variable ${ }^{11}$.

Se han documentado varias alteraciones citogenéticas asociadas al CRCC, siendo la hipoploidia y las alteraciones numéricas o estructurales del cromosoma 1 y de los autosomas las más frecuentemente observadas ${ }^{8}$.

El tumor que presentamos comparte hallazgos histológicos e inmunohistoquímicos típicos de los CRCC clásicos, sin embargo, presenta ciertas características propias de los CRCC de bajo grado publicadas por otros autores ${ }^{7,9}$, como son la ausencia de reacción desmoplásica, de atípia citológica en el epitelio de los conductos colectores vecinos y la escasa capacidad mitótica.

Radiológicamente suelen ser tumores de localización central con preservación del contorno renal, hipovasculares y mínimo realce con contraste. No hay duda de la eficacia de la nefrectomía radical en el tratamiento de los CRCC, aunque se han descrito con éxito nefrectomías parciales en el tratamiento de este tipo de tumores ${ }^{3}$. Con respecto al tratamiento adyuvante, no hay uno claramente establecido, pero parece ser que tanto la quimioterapia como la inmunoterapia no han ofrecido los beneficios esperados en términos de aumento de la supervivencia ${ }^{3-5}$.

\section{REFERENCIAS}

1. Herrera J, Caravaca F, Castaño JL, Soler J, Asuar S. Hipernefroma en riñón nativo de paciente con trasplante renal. Actas Urol Esp 2003;27(5):391-393.

2. Stewart JH, Buccianti G, Agodoa L, Gellert R, McCredie MR, Lowenfels $\mathrm{AB}$ et al. Cancers of the kidney and Urinary Tract in Patients on Dialysis for End-Stage Renal Disease: Analysis of Data from the United States, Europe, and Australia and New Zealand. J Am Soc Nephrol 2003;14(1):197-207. 
3. San Miguel P, Dos Santos JE, Delgado C, Zungri E, Álvarez C, Iglesias B. Carcinoma renal de los conductos colectores de bajo grado. Presentación de un caso y revisión de la literatura. Actas Urol Esp. 2004;28(6):478-483.

4. De Pablo A, Pinos MA, Jiménez JI, Jiménez JM, Lozano F, Rivas A y col. Carcinoma de ductos colectores de Bellini. Arch Esp Urol. 2004;57(2):153-156.

5. De la Peña E, Moreno J, Bocardo G, Redondo E, Silmi A, Aguilar E y col. Carcinoma de los conductos colectores. Experiencia personal y revisión de la literatura. Arch Esp Urol. 2002;55(3):275283

6. Fleming S, Lewi HJE. Collecting duct carcinoma of the kidney. Histopathology 1986;10(11):1131-1141.

7. Vélez MD, Blasco A, Iscar T, Herranz LM, Nieto S. Carcinoma de bajo grado de conductos colectores. Rev Esp Patol. 2004;37(3): 325-328.

8. Antonelli A, Portesi E, Cozzoli A, Zanotelli T, Tardanico R, Balzarini $\mathrm{P}$, et al. The collecting duct carcinoma of the kidney: a cytogenetical study. Eur Urol. 2003;43(6):680-685.
9. MacLennan GT, Farrow GM, Bostwick DG. Low-grade collecting duct carcinoma of the kidney: Report of 13 cases of lowgrade mucinous tubulocystic renal carcinoma of possible collecting duct origin. Urology 1997;50(5):679-684.

10. Daniel L, Zattara-Cannoni H, Lechevallier E, Pellissier JF. Association of a renal papillary carcinoma with a low grade tumour of the collecting ducts. J Clin Pathol. 2001;54(8):637-639.

Correspondencia autor: Dr. S. Martín Martín Servicio de Urología. Hospital Clínico Universitario Ramón y Cajal, s/n - 47005 Valladolid Tel.: 983420000

E-mail autor: risimartin7@hotmail.com Información artículo: Notas clínicas Trabajo recibido: noviembre 2006 Trabajo aceptado: noviembre 2006 